\title{
The effects of nonextensivity on quantum dissipation
}

SUBJECT AREAS:

THEORETICAL PHYSICS

QUANTUM MECHANICS

Received

10 September 2013

Accepted

13 January 2014

Published

28 January 2014

Correspondence and requests for materials should be addressed to

J.R.C. (choiardor@ hanmail.net)
Jeong Ryeol Choi

Department of Radiologic Technology, Daegu Health College, Yeongsong-ro 15, Buk-gu, Daegu 702-722, Republic of Korea.

Nonextensive dynamics for a quantum dissipative system described by a Caldirola-Kanai (CK) Hamiltonian is investigated in $\mathrm{SU}(1,1)$ coherent states. To see the effects of nonextensivity, the system is generalized through a modification fulfilled by replacing the ordinary exponential function in the standard CK Hamiltonian with the q-exponential function. We confirmed that the time behavior of the system is somewhat different depending on the value of $q$ which is the degree of nonextensivity. The effects of $q$ on quantum energy dissipation and other parameters are illustrated and discussed in detail.

$\mathrm{n}$ spite of the great success of Bolzmann-Gibbs statistics in describing statistical features of dynamical systems, it turned out that this statistics is inadequate in some cases for systems that follow nonextensive dynamics. Nonextensive systems are usually relevant to one or more phenomena among long-range spatial and/or temporal interactions, microscopic memory effects, multifractal space-time structure, anomalous diffusion, and self-gravitation ${ }^{1}$. This required an important amendment to thermostatistics so that the effects of nonextensivity are considered. A statistical mechanics proposed by Tsallis $^{2}$ is a powerful formalism to handle such systems. Numerous systems such as DNA biology ${ }^{3}$, multifractal space-time structures involved in dissipative systems $s^{4,5}$, and nonlinear phenomena in Euler turbulence ${ }^{6}$ are analyzed on the basis of Tsallis nonextensive thermostatistics ${ }^{2}$.

Recently, a research for the effects of nonextensivity on a generalized Caldirola-Kanai (CK) Hamiltonian system in $\mathrm{SU}(1,1)$ coherent state has been carried out by Özeren ${ }^{4}$. In fact, the problem of SU $(1,1)$ coherent state for dissipative systems has long been studied by many researchers ${ }^{7-12}$, mainly on the basis of CK Hamiltonian ${ }^{13,14}$. Özeren replaced the usual exponential function in the CK Hamiltonian with the q-exponential function which is crucial in nonextensive thermostatistics, and then investigated the time evolution of $\mathrm{SU}(1,1)$ coherent state parameters of the modified system.

The q-exponential function is useful for describing non-exponentially decaying or dissipating characteristics of physical systems such as fluorescence decays in complex biological systems ${ }^{15}$, surface potential decays of dielectric materials ${ }^{16}$, and spontaneous decays of unstable quantum systems ${ }^{17}$. Other types of non-exponentially decaying systems, that do not follow q-exponential decay, also exist. Some of them are the decay of frequency stable biophoton signals ${ }^{18,19}$ and the decay of the scalar field in the early universe $e^{20}$.

The purpose of this paper is to improve the previous theory ${ }^{4}$ of the nonextensive dynamics of a modified CK Hamiltonian system and to investigate its corresponding quantum behaviors in $\operatorname{SU}(1,1)$ coherent state on the basis of the improved theory. To this end, an exact Hamiltonian dynamics will be employed and a semiclassical behavior of the time evolution of $\mathrm{SU}(1,1)$ coherent state parameter and energy dissipation will be examined.

This paper is organized in the following order. First, a modified CK Hamiltonian that describes dissipation is introduced and the corresponding SU $(1,1)$ generators are established. Then, the effects of nonextensivity on the $\mathrm{SU}(1,1)$ coherent states of the system are investigated and the corresponding results are compared to those of the previous work ${ }^{4}$. Finally, nonextensive dynamics of the system is discussed and concluding remarks are given.

\section{Results}

Description of the generalized CK Hamiltonian. There has been great concern for dynamical problems of dissipative systems in both classical and quantum points of view for several decades (see, for example, Ref. 21 and references there in). However, most research in this direction is focused on the damped system described by standard CK oscillator. The generalization of the CK Hamiltonian system regarding nonextensive dynamics may offer interesting consequences that are absent in the standard one. Here, we introduce $S U(1,1)$ generators for the generalized CK Hamiltonian system and show how to describe it in terms of SU(1,1) generators. 
We start with the standard CK Hamiltonian that is given by ${ }^{13,14,22,23}$

$$
\hat{H}=e^{-\lambda t} \frac{\hat{p}^{2}}{2 m}+\frac{1}{2} e^{\lambda t} m \omega^{2} \hat{q}^{2},
$$

where $\lambda$ is a damping constant. As you can see, this Hamiltonian is just obtained by replacing the mass of the simple harmonic oscillator Hamiltonian such that

$$
m \rightarrow m e^{\lambda t} .
$$

Let us now focus on the exponential function given in this expression. A more generalized exponential function, so-called the q-exponential function, that is necessary in nonextensive mechanics is defined $\mathrm{as}^{24}$

$$
\exp _{\mathbf{q}}(z)=[1+(1-\mathbf{q}) z]^{1 /(1-\mathbf{q})}
$$

under the condition $1+(1-\mathbf{q}) z \geq 0$. If $\mathbf{q}<1$, the system is superextensive whereas it is sub-extensive when $\mathbf{q}>1$. This function corresponds to a non-equilibrium distribution function and is equivalent to the Zipf-Mandelbrot distribution in the case that $\mathbf{q}$ is larger than unity. It reverts to the original exponential function when $q=1$. Notice that there appears a power-law tail in this function in an asymptotic limit ${ }^{25}$.

The nonextensive thermostatistics uses the nonadditive Tsallis entropy and after the standard maximization procedure under appropriate constraints, the q-exponentials and q-Gaussians appear as the probability densities. Two possible choices in the maximization procedure are those that use either standard mean or escort mean. If the escort mean is used, the definition of the q-exponential permits us to represent its inverse function as $\exp _{\mathbf{q}}(-z)$ with $\mathbf{q}>1$, as already used in Ref. 4. However, if the standard mean is used, the definition of the q-exponential does not allow us to replace $\exp _{\mathbf{q}}$ $(z)]^{-1}$ by $\exp _{\mathbf{q}}(-z)$ in the range $\mathbf{q}<1^{26}$. One can easily confirm this fact from Fig. 1. We will adopt standard mean in this work in order to investigate the nonextensive characteristics of the generalized dissipative system.

The generalized CK Hamiltonian is obtained by replacing ordinary exponential function with $\mathbf{q}$-exponential one such that

$$
\hat{H}_{\mathbf{q}}=\frac{\hat{p}^{2}}{2 m \exp _{\mathbf{q}}(\lambda t)}+\frac{1}{2} \exp _{\mathbf{q}}(\lambda t) m \omega^{2} \hat{q}^{2} .
$$

Many quantum features for a family of CK Hamiltonian systems, including phase coherence, quantum tunneling, and quantum fluctuations, can be studied using SU $(1,1)$ Lie algebra ${ }^{27}$. From Methods section (A), we can confirm that Eq. (4) can be rewritten as

$$
\hat{H}_{\mathbf{q}}=2 \hbar \omega \cosh _{\mathbf{q}}(\lambda t) \hat{K}_{0}+\hbar \omega \sinh _{\mathbf{q}}(\lambda t)\left(\hat{K}_{+}+\hat{K}_{-}\right),
$$

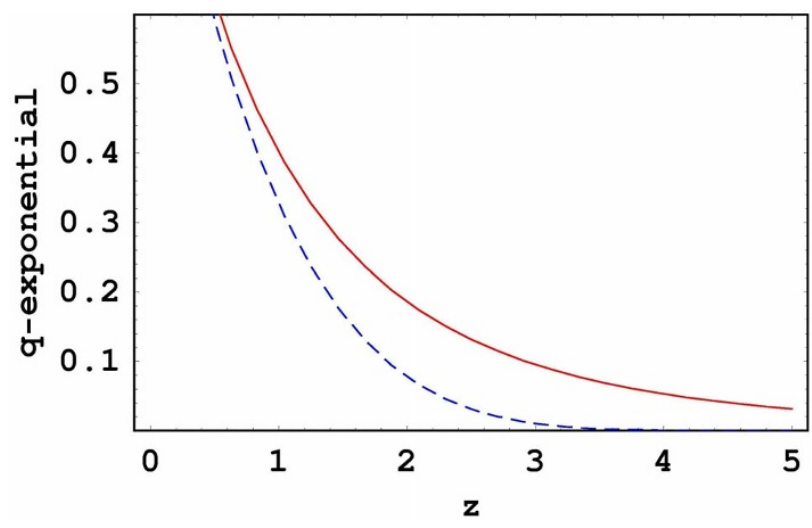

Figure $1 \mid$ Comparison of $\left[\exp _{\mathrm{q}}(z)\right]^{-1}$ (solid red line) with $\exp _{\mathrm{q}}(-z)$ (dashed blue line) for $\mathrm{q}=0.8$. where $\hat{K}_{0}, \hat{K}_{+}, \hat{K}_{-}$are $\mathrm{SU}(1,1)$ generators defined in Eqs. (21) and (24) and deformed hyperbolic trigonometric functions are given by

$$
\begin{aligned}
& \cosh _{\mathbf{q}}(z)=\frac{1}{2}\left\{\exp _{\mathbf{q}}(z)+\left[\exp _{\mathbf{q}}(z)\right]^{-1}\right\}, \\
& \sinh _{\mathbf{q}}(z)=\frac{1}{2}\left\{\exp _{\mathbf{q}}(z)-\left[\exp _{\mathbf{q}}(z)\right]^{-1}\right\} .
\end{aligned}
$$

We will use this Hamiltonian in order to investigate the effects of nonextensivity on the CK Hamiltonian system. One can easily confirm that Eq. (5) is somewhat different from that of previous reports (Ref. 4).

Time behavior of the system. Now, the characteristics of $\mathrm{SU}(1,1)$ coherent state for the generalized CK Hamiltonian system described by Eq. (5) will be investigated. Among several classes of the SU $(1,1)$ coherent state, we are interested in the Perelomov coherent state ${ }^{28}$. If we introduce the displacement operator of the form

$$
\hat{D}(\alpha)=\exp \left[\frac{1}{2}\left(\alpha^{2} \hat{K}_{+}-\alpha^{* 2} \hat{K}_{-}\right)\right],
$$

where $\alpha$ is an eigenvalue of the operator $\hat{a}$ that is defined as

$$
\hat{a}=[m \omega /(2 \hbar)]^{1 / 2} \hat{q}+(2 m \omega \hbar)^{-1 / 2} \hat{p},
$$

the Perelomov coherent state is obtained by acting $\hat{D}(\alpha)$ on vacuum state, such that

$$
|\zeta ; k\rangle=\hat{D}(\alpha)|0\rangle_{k} .
$$

Of course, $\hat{a}$ and its Hermitian adjoint satisfy the well known commutation relation: $\left[\hat{a}, \hat{a}^{\dagger}\right]=1$. We also introduce a $\operatorname{SU}(1,1)$ coherent state parameter $\zeta$, which is

$$
\zeta=\frac{\alpha^{2}}{|\alpha|^{2}} \tanh \left(|\alpha|^{2} / 2\right)
$$

We can see the time evolution of any physical observables from their expectation values. For the case of the Hamiltonian, Eq. (5), the expectation value in the Perelomov coherent state is obtained from

$$
H_{\mathbf{q}, k}=\left\langle\zeta ; k\left|\hat{H}_{\mathbf{q}}\right| \zeta ; k\right\rangle \text {. }
$$

Considering the formulae given in Eqs. (27)-(29) in the Methods section, we easily have

$$
H_{\mathbf{q}, k}=\frac{2 \hbar \omega k}{1-|\zeta|^{2}}\left[\left(1+|\zeta|^{2}\right) \cosh _{\mathbf{q}}(\lambda t)+\left(\zeta+\zeta^{*}\right) \sinh _{\mathbf{q}}(\lambda t)\right] .
$$

Notice that this varies depending on time. To identify the complete time behavior of this quantity, it is necessary to know the explicit form of $\zeta$. For this reason, we see a semiclassical behavior of the time evolution of $\zeta$. Let us separate $\zeta(t)$ into real and imaginary parts such that $\zeta(t)=x(t)+i y(t)$ where $x$ and $y$ are real. Then, by inserting this into Eq. (32) given in the Methods section, we have the formula for the time evolution of each parts

$$
\begin{gathered}
\dot{x}=2 \omega\left[y \cosh _{\mathbf{q}}(\lambda t)+x y \sinh _{\mathbf{q}}(\lambda t)\right], \\
\dot{y}=-\omega\left[2 x \cosh _{\mathbf{q}}(\lambda t)+\left(1+x^{2}-y^{2}\right) \sinh _{\mathbf{q}}(\lambda t)\right] .
\end{gathered}
$$

The parametric plot of $(x(t), y(t))$ is given in Fig. 2 with different initial conditions $(x(0), y(0))$. Notice that this is very different from that of the previous report ${ }^{4}$. For the convenience of comparison, we have taken all auxiliary values to be the same as those of Ref. 4 . The 


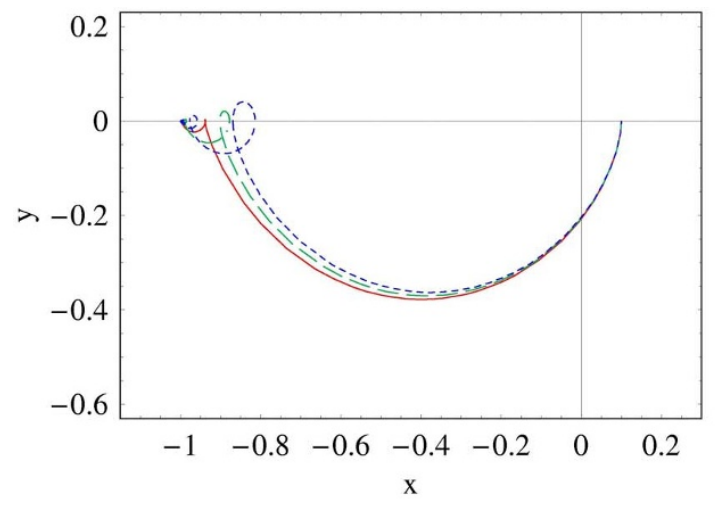

(a)

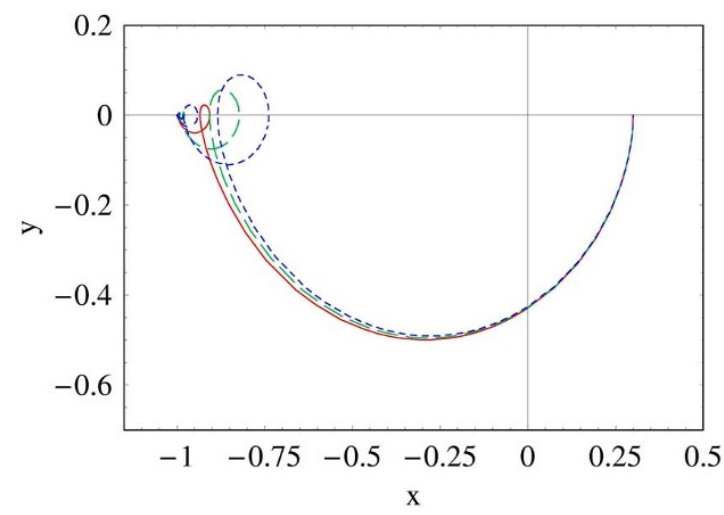

(b)

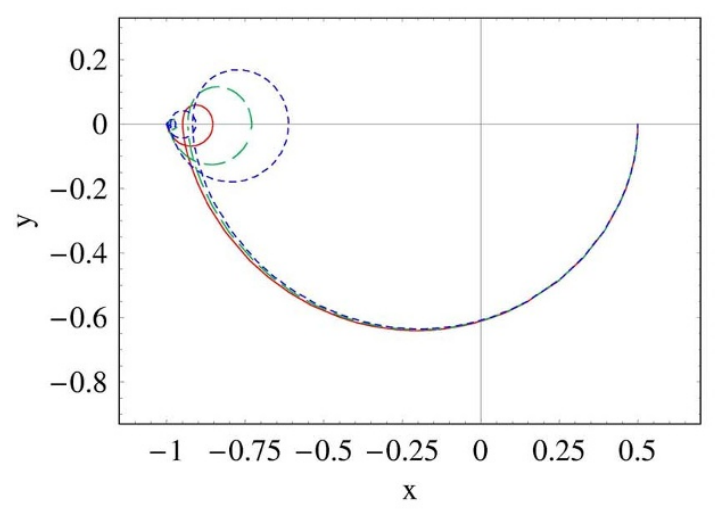

(c)

Figure $2 \mid$ Parametric plot of $\zeta(t)$ (i.e., $x(t)$ and $y(t)$ ) with the use of Eqs. (14) and (15). The initial condition $(x(0), y(0))$ is $(0.1,0)$ for $(a),(0.3,0)$ for (b), and $(0.5,0)$ for (c). The value of $\mathbf{q}$ is 1.0 for solid red line, 0.9 for long dashed green line and 0.8 for short dashed blue line. We used $\omega=1$ and $\lambda$ $=1$. The range of plot time is $\left(t_{\text {initial }}, t_{\text {final }}\right)=(0,15)$.

trajectory of $(x(t), y(t))$ in that work approaches $(1,0)$ as time goes by. However, $(x(t), y(t))$ in this work follows quite a different trajectory from that and ends up with $(x(t), y(t))=(-1,0)$ for large $t$. This discrepancy partly originates from the statistical mean, i.e., we used standard mean while escort mean is used in Ref. 4. However, the contribution of such different choices of statistical mean on the discrepancy is minor. The main difference between them stems from the misderivation of the Hamiltonian in that work.

Now we investigate the nonextensive effects on time evolution of the system on the basis of the improved theory described so far. To do this, we consider the dynamical energy of the system and the fluctuations of the canonical variables. In terms of $x$ and $y$, Eq. (13) can be rewritten as

$$
H_{\mathbf{q}, k}=\frac{2 \hbar \omega k}{1-x^{2}-y^{2}}\left[\left(1+x^{2}+y^{2}\right) \cosh _{\mathbf{q}}(\lambda t)+2 x \sinh _{\mathbf{q}}(\lambda t)\right] .
$$

The energy of the system is in general different from the Hamiltonian for dissipative systems ${ }^{29}$. In this case, the quantum energy is given by

$$
E_{\mathbf{q}, k}=\left[\exp _{\mathbf{q}}(\lambda t)\right]^{-1} H_{\mathbf{q}, k}
$$

From Fig. 3, we see that the quantum energy decreases with time due to dissipation. The energy dissipates more rapidly for large $\mathbf{q}$ due to the effects of nonextensivity. The rate of energy dissipation with time is locally maximum when the velocity of the oscillator is largest in the vicinity of the point $q=0$. On the other hand, the energy does not dissipate instantaneously at the turning point of the oscillator. This outcome agrees with that of the classical analysis.

Let us see the fluctuations of canonical variables. The fluctuation of an arbitrary observable $\hat{\mathcal{O}}$ is defined in the form

$$
\Delta \mathcal{O}=\left[\left\langle\zeta ; k\left|\hat{\mathcal{O}}^{2}\right| \zeta ; k\right\rangle-\langle\zeta ; k|\hat{\mathcal{O}}| \zeta ; k\rangle^{2}\right]^{1 / 2} \text {. }
$$

According to this, we can obtain the fluctuation of canonical variables to be

$$
\begin{gathered}
\Delta q=\sqrt{\frac{2 \hbar k}{m \omega\left(1-x^{2}-y^{2}\right)}}\left(1+2 x+x^{2}+y^{2}\right)^{1 / 2}, \\
\Delta p=\sqrt{\frac{2 m \omega \hbar k}{1-x^{2}-y^{2}}}\left(1-2 x+x^{2}+y^{2}\right)^{1 / 2} .
\end{gathered}
$$

From Fig. 4, we confirm that both $\Delta q$ and $\Delta p$ oscillate with time. The envelope of oscillation for $\Delta q$ decreases with time whereas that for $\Delta p$ increases. The time variation of $\Delta q$ and $\Delta p$ increases as $\mathrm{q}$ grows as a consequence of nonextensive dynamics. We can confirm from Fig. 4 (c) that the uncertainty product $\Delta q \Delta p$ also varies significantly with time. However, $\Delta q \Delta p$ is always larger than (or equal to) $\hbar / 2$ so that the uncertainty principle holds.

\section{Discussion}

The effects of nonextensivity on time evolution of the generalized CK Hamiltonian system that reveals dissipation are investigated through $\mathrm{SU}(1,1)$ coherent state description. Using a correct Hamiltonian

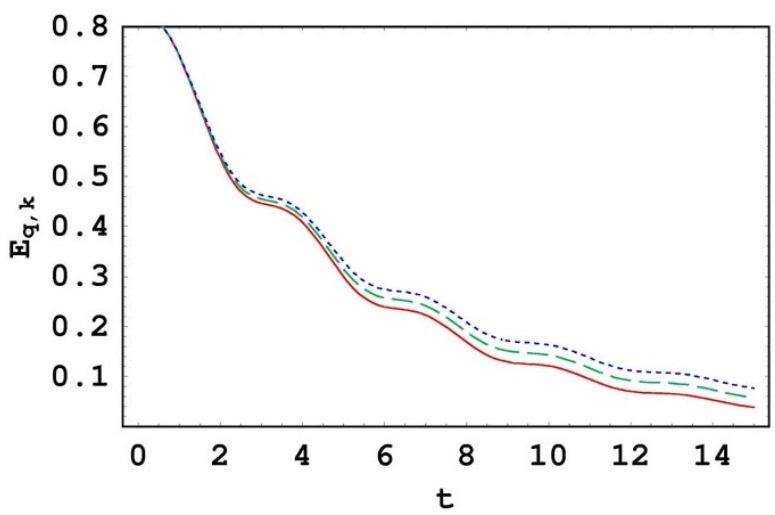

Figure $3 \mid$ The energy expectation value $E_{\mathrm{q}, \boldsymbol{k}}$. The value of $\mathbf{q}$ is 1.0 for solid red line, 0.9 for long dashed green line and 0.8 for short dashed blue line. We used $\omega=1, \lambda=0.2, \hbar=1$, and $k=1 / 4$. 


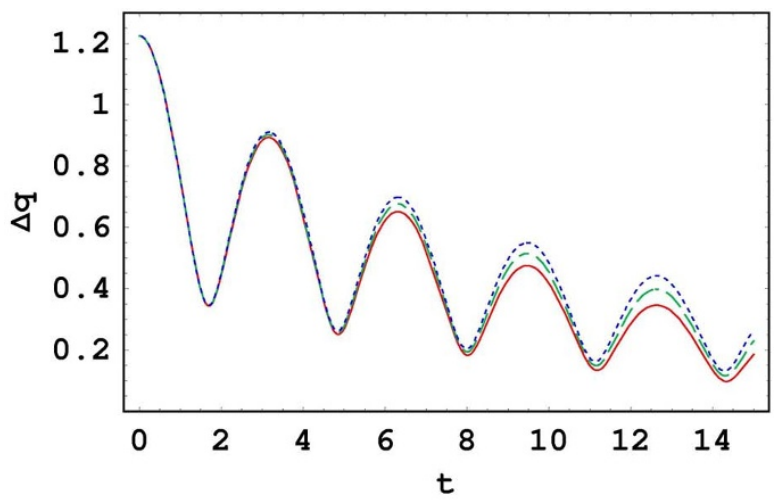

(a)

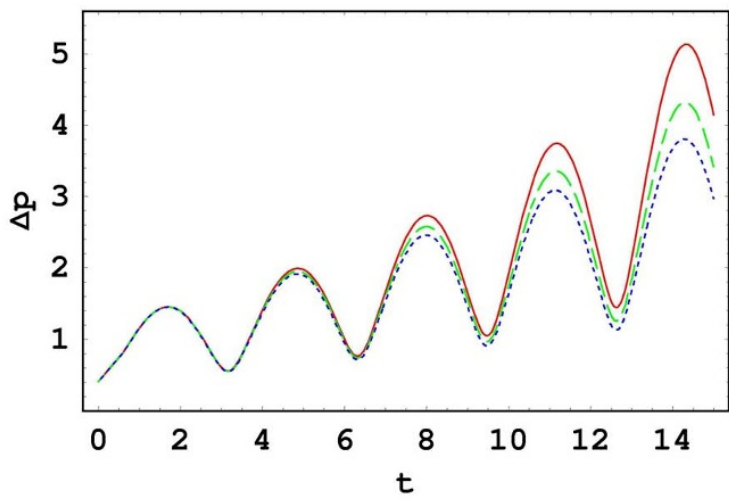

(b)

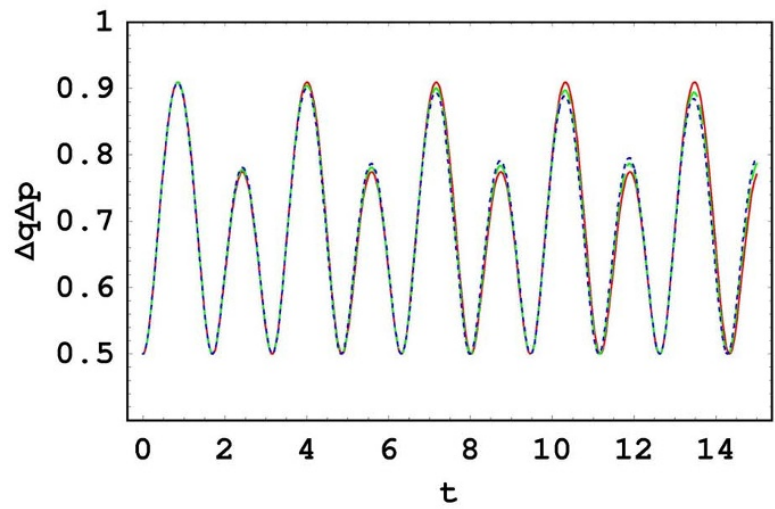

(c)

Figure $4 \mid$ The fluctuations $[\Delta q(a)$ and $\Delta p(b)]$ and uncertainty product $\Delta q \Delta p(\mathrm{c})$. The value of $q$ is 1.0 for solid red line, 0.9 for long dashed green line and 0.8 for short dashed blue line. We used $\omega=1, \lambda=0.2, \hbar=1, m=$ 1 , and $k=1 / 4$.

formalism, we improved the existing theory ${ }^{4}$ for its nonextensive dynamics. To construct the Hamiltonian of the system, ordinary exponential function in CK Hamiltonian is replaced by q-exponential function. From the parametric plot of $S U(1,1)$ coherent state parameter $\zeta(t)$, the trajectory associated with its real and imaginary parts, $(x(t), y(t))$, is investigated and, as a result, we have shown that it is different depending on the value of the nonextensive parameter $\mathbf{q}$ (see Fig. 2).

We further investigated the time evolution of the system on the basis of our improved theory. It is shown in Fig. 3 that the quantum energy $E_{\mathbf{q}, k}$ decreases with time like a pure classical one. The quantum energy dissipates rapidly when the oscillator attains highest velocity at the vicinity of origin $(q=0)$ whereas it does not dissipates at the turning point of the oscillator. For a better understanding of this, recall that the ratio of energy dissipation with time for a damped system is proportional to the velocity of the mass. This consequence obtained in quantum domain cannot be deduced from the existing theory of Ref. 4 and agrees well with the classical theory. Quantum and classical correspondence is important in order that a certain quantum theory is valid.

The fluctuations $\Delta q$ and $\Delta p$ oscillate with time: the envelope of the oscillation of $\Delta q$ decreases with time while that of $\Delta p$ increases. Moreover, the uncertainty product $\Delta q \Delta p$ varies significantly with time. As an appearance of nonextensive effects, the time variation of not only $E_{\mathbf{q}, k}$ but also $\Delta q, \Delta p$, and $\Delta q \Delta p$ became large as $\mathbf{q}$ increases. Such analysis is exact and different from the one predicted from the theory of previous reports ${ }^{4}$. The outcomes of this research may serve as important consequences, which are absent in the standard one that is not considered nonextensivity, on predicting time behavior of physical quantities of the system depending on the value of $\mathbf{q}$. For $\mathbf{q} \rightarrow 1$, our results recover to the ordinary ones that correspond to the extensive system ${ }^{11}$. Indeed, the q-exponential function is ubiquitous and plays a crucial role in the diverse branches of physical systems that exhibit nonextensive character.

\section{Methods}

(A) SU $(1,1)$ generators and the modified CK Hamiltonian. We introduce $\mathrm{SU}(1,1)$ generators of the form

$$
\begin{gathered}
\hat{K}_{0}=\frac{1}{4 \hbar}\left(m \omega \hat{q}^{2}+\frac{\hat{p}^{2}}{m \omega}\right), \\
\hat{K}_{1}=\frac{1}{4 \hbar}\left(m \omega \hat{q}^{2}-\frac{\hat{p}^{2}}{m \omega}\right), \\
\hat{K}_{2}=-\frac{1}{4 \hbar}[\hat{q} \hat{p}+\hat{p} \hat{q}] .
\end{gathered}
$$

In terms of these, the annihilation and the creation operators are given by

$$
\hat{K}_{-}=\hat{K}_{1}-i \hat{K}_{2}, \quad \hat{K}_{+}=\hat{K}_{1}+i \hat{K}_{2} .
$$

Now it is possible to represent the square of position and momentum operators in the from

$$
\begin{aligned}
& \hat{q}^{2}=\frac{\hbar}{m \omega}\left(2 \hat{K}_{0}+\hat{K}_{+}+\hat{K}_{-}\right), \\
& \hat{p}^{2}=m \omega \hbar\left(2 \hat{K}_{0}-\hat{K}_{+}-\hat{K}_{-}\right) .
\end{aligned}
$$

If we consider these equations, the Hamiltonian given in Eq. (4) can be rewritten in terms of $\mathrm{SU}(1,1)$ generators to be Eq. (5).

Finally, the formulae necessary for evaluating Eq. (12) are given by ${ }^{30,31}$

$$
\begin{aligned}
& \left\langle\zeta ; k\left|\hat{K}_{0}\right| \zeta ; k\right\rangle=k \frac{1+|\zeta|^{2}}{1-|\zeta|^{2}}, \\
& \left\langle\zeta ; k\left|\hat{K}_{+}\right| \zeta ; k\right\rangle=\frac{2 k \zeta^{*}}{1-|\zeta|^{2}}, \\
& \left\langle\zeta ; k\left|\hat{K}_{-}\right| \zeta ; k\right\rangle=\frac{2 k \zeta}{1-|\zeta|^{2}} .
\end{aligned}
$$

(B) Time evolution of the $\mathrm{SU}(1,1)$ coherent state parameter. The Euler-Langrange equation that describes the classical equation of motion for $\zeta$ introduced in Eq. (11) is given $b^{4}$

$$
\dot{\zeta}=\left[\zeta, H_{\mathbf{q}, k}\right] /(i \hbar),
$$

where $\left[C_{1}, C_{2}\right]$ is a generalized Poisson bracket: 


$$
\left[C_{1}, C_{2}\right]=\frac{\left(1-|\zeta|^{2}\right)^{2}}{2 k}\left(\frac{\partial C_{1}}{\partial \zeta} \frac{\partial C_{2}}{\partial \zeta^{*}}-\frac{\partial C_{1}}{\partial \zeta^{*}} \frac{\partial C_{2}}{\partial \zeta}\right) .
$$

The execution of a little algebra after substituting Eq. (13) into Eq. (30) yields

$$
\dot{\zeta}=-i \omega\left[2 \zeta \cosh _{\mathbf{q}}(\lambda t)+\left(1+\zeta^{2}\right) \sinh _{\mathbf{q}}(\lambda t)\right] .
$$

1. Tsallis, C. Introduction to Nonextensive Statistical Mechanics, Springer-Verlag, New York, 2009.

2. Tsallis, C. Possible generalization of Boltzmann-Gibbs statistics. J. Stat. Phys. 52, 479-487 (1988).

3. Oikonomou, Th., Provata, A. \& Tirnakli, U. Nonextensive statistical approach to non-coding human DNA. Physica A 387, 2653-2659 (2008).

4. Özeren, S. F. The effect of nonextensivity on the time evolution of the SU(1,1) coherent states driven by a damped harmonic oscillator. Physica A 337, 81-88 (2004).

5. Varela, L. M., Carrete, J., Muñoz-Solá, R., Rodríguez, J. R. \& Gallego, J. Nonextensive statistical mechanics of ionic solutions. Phys. Lett. A 370, 405-412 (2007).

6. Boghosian, B. M. Thermodynamic description of the relaxation of twodimensional turbulence using Tsallis statistics. Phys. Rev. E 53, 4754-4763 (1996).

7. Ban, M. SU $(1,1)$ Lie algebraic approach to linear dissipative processes in quantum optics. J. Math. Phys. 33, 3213-3228 (1992).

8. Obada, A.-S. F., Hessian, H. A. \& Mohamed, A.-B. A. Output entanglement from $\mathrm{SU}(1,1)$ coherent states under nonlinear dissipation in the dispersive limit. J. Phys. A.: Math. Theor. 43, 025305 (2010).

9. Blasone, M. \& Jizba, P. Bateman's dual system revisited: quantization, geometric phase and relation with the ground-state energy of the linear harmonic oscillator. Ann. Phys. 312, 354-397 (2004).

10. Choi, J. R. \& Choi, S. S. SU(1,1) Lie algebra applied to the time-dependent quadratic Hamiltonian system perturbed by a singularity. Int. J. Mod. Phys. B 18, 3429-3441 (2004).

11. Choi, J. R. \& Yeon, K. H. Dynamics of SU $(1,1)$ coherent states for the damped harmonic oscillator. Phys. Rev. A 79, 054103(1-4) (2009).

12. Gerry, C. C., Ma, P. K. \& Vrscay, E. R. Dynamics of SU $(1,1)$ coherent states driven by a damped harmonic oscillator. Phys. Rev. A 39, 668-674 (1989).

13. Caldirola, P. Forze non conservative nella meccanica quantistica. Nuovo Cimento 18, 393-400 (1941).

14. Kanai, E. On the quantization of the dissipative systems. Prog. Theor. Phys. 3, 440-442 (1948).

15. Wlodarczyk, J. \& Kierdaszuk, B. A new approach to interpretation of heterogeneity of fluorescence decay in complex biological systems. Progress in Biomedical Optics and Imaging - Proceedings of SPIE 5862, 58620X(1-8) (2005).

16. Horikawa, J. \& Wada, T. Experimental investigation on the surface potential decays of dielectric materials with q-exponential function. Journal of Physics: Conference Series 201, 012010(1-7) (2010).

17. Wilk, G. \& Wodarczyk, Z. Nonexponential decays and nonextensivity. Phys. Lett. A 290, 55-58 (2001).
18. Bajpai, R. P., Kumar, S. \& Sivadasan, V. A. Biophoton emission in the evolution of a squeezed state of frequency stable damped oscillator. Appl. Math. Comput. 93, 277-288 (1998).

19. Choi, J. R. Thermal radiation fields in time-dependent linear media at finite temperature. Laser Phys. Lett. 10, 105202(1-8) (2013).

20. Choi, J. R. SU(1,1) Lie algebraic approach for the evolution of the quantum inflationary universe. Physics of the Dark Universe 2, 41-49 (2013).

21. Um, C.-I., Yeon, K.-H. \& George, T. F. The quantum damped harmonic oscillator. Phys. Rep. 362, 63-192 (2002).

22. Dodonov, V. V. \& Man'ko, V. I. Coherent states and the resonance of a quantum damped oscillator. Phys. Rev. A 20, 550-560 (1979).

23. Yeon, K. H., Um, C. I. \& George, T. F. Coherent states for the damped harmonic oscillator. Phys. Rev. A 36, 5287-5291 (1987).

24. Tsallis, C. What are the numbers that experiments provide? Quimica Nova 17, 468-471 (1994).

25. Yamada, H. S. \& Iguchi, K. q-exponential fitting for distributions of family names. Physica A 387, 1628-1636 (2008).

26. Oikonomou, T. \& Bagci, G. B. The maximization of Tsallis entropy with complete deformed functions and the problem of constraints. Phys. Lett. A 374, 2225-2229 (2010).

27. Wódkiewicz, K. \& Eberly, J. H. Coherent states, squeezed fluctuations, and the $\mathrm{SU}(2)$ and $\mathrm{SU}(1,1)$ groups in quantum-optics applications. J. Opt. Soc. Am. B 2, 458-466 (1985).

28. Perelomov, A. M. Coherent states for arbitrary Lie group. Commun. Math. Phys. 26, 222-236 (1972).

29. Marchiolli, M. A. \& Mizrahi, S. S. Dissipative mass-accreting quantum oscillator. J. Phys. A: Math. Gen 30, 2619-2635 (1997).

30. Gerry, C. C. \& Silverman, S. Path integral for coherent states of the dynamical group SU(1,1). J. Math. Phys. 23, 1995-2003 (1983).

31. Gerry, C. C. Conserved noether currents and path integrals for dynamical groups. Phys. Lett. B 119, 381-384 (1982).

\section{Acknowledgments}

This research was supported by the Basic Science Research Program through the National Research Foundation of Korea (NRF) funded by the Ministry of Education (Grant No.: NRF-2013R1A1A2062907).

\section{Author contributions}

J.R.C. wrote the paper and approved it.

\section{Additional information}

Competing financial interests: The authors declare no competing financial interests.

How to cite this article: Choi, J.R. The effects of nonextensivity on quantum dissipation. Sci. Rep. 4, 3911; DOI:10.1038/srep03911 (2014).

(c) (i) $\Theta$ This work is licensed under a Creative Commons AttributionBY No No Nommercial-NoDerivs 3.0 Unported license. To view a copy of this license, visit http://creativecommons.org/licenses/by-nc-nd/3.0 